\title{
An Asymmetric Watermarking Method in the DCT Domain Based on RC4-Permutation and Chaotic Map
}

\author{
${ }^{1}$ Rinaldi Munir, ${ }^{1}$ Bambang Riyanto, ${ }^{1}$ Sarwono Sutikno \& ${ }^{2}$ Wiseto P. Agung \\ ${ }^{1}$ Bandung Institute of Technology, Jl. Ganesha 10, Bandung 40132, Indonesia \\ ${ }^{2} \mathrm{PT}$. Telekomunikasi Indonesia, Jl. Gegerkalong, Bandung, Indonesia
}

\begin{abstract}
This paper presents an asymmetric watermarking method in the DCT domain for still images based on permutation and chaos. This method uses secret watermark as private key and public watermark as public key. The public watermark has a normal distribution with mean $=0$ and variance $=1$. The secret watermark is obtained by permutating the public watermark according to combination of a part of RC4 algorithm and a logistic map. The watermark is embedded into mid-frequency components of the DCT block for better robustness. The detection process is implemented by correlation test between the public watermark and the received image. Experiments show that the watermarking method was proved to be robust againts some typical image processings (cropping, JPEG compression, resizing, rotation, sharpening, and noising).
\end{abstract}

Keywords: asymmetric; chaos; correlation; DCT; image; RC4; robust; watermarking.

\section{Introduction}

Recently, with the emergence of computer network and Internet, lots of digital multimedia data are easily copied, stored and transmitted over the world, leading to illegal copy or unauthorized use. Digital watermarking has been used widely as a tool for protecting copyright of digital multimedia data (e.g. images) [1]. Basic requirements of a digital watermarking scheme are imperceptibility, robustness, and security [2]. A watermark is inserted into digital images so that it is imperceptible to a person. The watermark must be robust to typical signal processing operations such as JPEG compression, cropping, resizing, noising, rotation, and so on. The watermark should also only be accessible by authorized parties.

Many digital watermarking methods for still images have been proposed [1-3]. The particular problem with the state-of-the-art watermarking method is that the majority of these schemes are symmetric. The symmetric watermarking is similar to symmetric cryptography: the same key is used for watermark embedding and detection. The symmetric watermarking scheme has a security problem. In many watermarking methods, the secret key represents the watermark itself or specifies the embedding location of the watermark. Because 
the watermarking algorithm is published, once an attacker knows the secret key, the watermark can be detected, and in addition, be easily estimated and removed from the multimedia data completely without making any degradation and thereby defeats the goal of copyright protection.

A solution to solve the problem is an asymmetric watermarking scheme, in which different key(s) are used for watermark embedding and detection. An asymmetric watermarking system uses the secret key to embed a watermark and another key to verify the watermark. It is not necessary for detector to know the secret key. An asymmetric watermarking is called public-key watermarking if key used for detecting watermark is available publicly, so the key is called public key. Only the copyright holder has his/her private key to embed a watermark. Anybody who knows the public key could detect the watermark. Therefore, an asymmetric watermarking can provide public detection, but the secret key cannot be deduced from the public key. Also, knowing the public key does not enable an attacker to remove the watermark [3]. Asymmetric watermarking methods have been proposed in recent years. Review of several existing methods can be found in [4].

Generally, in asymmetric watermarking scheme, the secret key is a secret (or private) watermark embedded into host media and the public key is a public watermark. To enable detection, the public watermark should have a correlation with the secret watermark. The detection step is implemented by correlation test between public watermark and multimedia data received [5]. Comparing the detection value with a predefined threshold, a decision should be made to decide the presence of the embedded watermark.

There exist numerous methods to generate private watermarks that are different from but have fixed correlations with public watermarks. One of them is by using permutation. In this paper we present an asymmetric watermarking method based on permutation. We use a combination of a part of RC4 cipher and a chaotic map as permutation method. A chaotic map is used to produce a pseudo-random signal. In recent years, chaos has been used for digital watermarking to increase security [6], due to its sensitivity to initial condition. This method has high robustness, and it is secure to a malicious attack (the attack whose objective is removing the watermark from the watermarked image.

In comparison to existing related methods [7-8], in this paper we propose a combination of RC4-permutation and chaotic map for asymmetric watermarking method. In this method the watermark is embedded into the transform-domain (DCT domain). 
The rest of the paper is organized as follows. In Section 2 we will present chaotic function, in Section 3 we present RC4 algorithm, and in Section 4 we present watermarking in DCT domain. Next, in Section 5 we present the asymmetric proposed watermarking method, in Section 6 we report the experimental results, and finally some conclusions in Section 8.

\section{Chaos Function}

One of the characteristics of chaotic systems is a sensitivity to initial conditions; i.e. two relatively close initial value will diverge as the system evolves. As a result of this sensitivity, the behavior of systems appears to be random, even though the system is deterministic; i.e. it is well defined and contains no random parameters. Hence, a chaotic system can be used as a pseudo-random generator. It means a large number of non-periodic, noise-like yet deterministic and reproducible sequences can be generated [9].

We consider a 1-D discrete chaotic map $\mathbf{F}: U \rightarrow U, U \subset \mathbf{R}$, which provides a sequence of real number:

$$
x_{k+1}=\mathbf{F}\left(x_{k}, \lambda\right), x_{k} \in U, \lambda \in \mathbf{R}
$$

where $n=0,1,2, \ldots$ denotes map iterations and $\lambda$ is a parameter that controls the dynamic behavior of the chaotic map. In our scheme, an initial value of chaotic map behaves as the key of the watermarking system.

One of the simplest chaotic maps is a logistic map [6], which is a recurrence relation that describes population growth over time, described by

$$
x_{k+1}=\mu x_{k}\left(1-x_{k}\right)
$$

where $0 \leq \mu \leq 4$. When we iterate equation (2) from an initial value $\left(x_{0}\right)$, we get a chaotic sequence. Elements of this sequence are between 0 and 1 .

\section{$3 \quad$ Permutation Based on RC4}

RC4 (also known as ARC4 or ARCFOUR), one of cryptography algorithms (cipher), is the most popular stream cipher. RC4 generates a pseudorandom stream of bits (a keystream) that is combined with the plaintext using XOR (encryption) or with the ciphertext (decryption). This algorithm uses array $S[0.255]$ that is initialized with $0,1, \ldots, 255$. Before encryption/decryption process, elements of $S$ are permutated based on external key $U$. Length of $U$ is variable, but if the length of $U$ is less than 256 characters, we must do padding so that its length is equal to 256 characters. The algorithm for permutating elements of $S$ is as follows: 


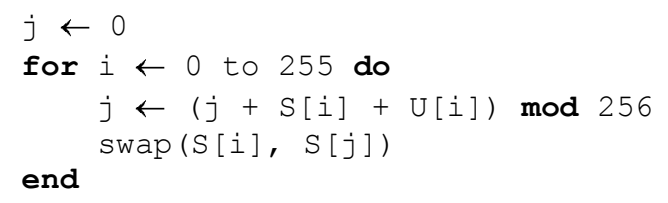

This algorithm is modified to permutate the public watermark. Suppose that the length of the watermark is $N$, then elements of $S$ are initialized with $0,1, \ldots, N$. Key $U$, with the length $N$, is an integer array whose elements are generated by a logistic map (each chaos value is multiplied by $N$ and then rounded to the nearest integer). Next, elements of $S$ are permutated with the algorithm except 255 is replaced by $N-1$. The values in $S$ are used to permutate the public watermark.

\section{$4 \quad$ Watermarking in DCT Domain}

Current image watermarking methods can be grouped into spatial domain methods and transform domain methods. In spatial domain, we embed the watermark by directly modifying the pixel values of the original image. In transform domain, a transformation is first applied to the original image and then embedding the watermark into transform coefficient. There are three main transform methods that are generally employed, i.e. Fourier transform (DFT), discrete cosine transform (DCT), and wavelet transform (DWT). Embedding the watermark into the transform-domain can increase the robustness, when the watermarked images are tested after having been subjected to common image processing. In this paper we transform the original image using DCT method. The DCT is chosen because it is simple and mainly for its assonance with JPEG coding standard.

The two-dimensional $D C T$ of an $M$ by $N$ matrix is defined as follows [2]:

$$
C(p, q)=\alpha_{p} \alpha_{q} \sum_{m=0}^{M-1} \sum_{n=0}^{N-1} I(m, n) \cos \frac{\pi 2(m+1) p}{2 M} \cos \frac{\pi(2 n+1) q}{2 N}
$$

and the inverse DCT (or IDCT) is given by

$$
I(m, n)=\sum_{m=0}^{M-1} \sum_{n=0}^{N-1} \alpha_{p} \alpha_{q} C(p, q) \cos \frac{\pi 2(m+1) p}{2 M} \cos \frac{\pi(2 n+1) q}{2 N}
$$

where 


$$
\alpha_{p}=\left\{\begin{array}{cc}
\frac{1}{\sqrt{M}} & , p=0 \\
\sqrt{\frac{2}{M}} & , 1 \leq p \leq M-1
\end{array} \quad \alpha_{q}=\left\{\begin{array}{cc}
\frac{1}{\sqrt{N}} & , q=0 \\
\sqrt{\frac{2}{N}} & , 1 \leq q \leq N-1
\end{array}\right.\right.
$$

The values $C(p, q)$ are called the DCT coefficients of image $I$.

The DCT can be applied to transform the whole image or image blocks $(8 \times 8$ pixel). By referring to JPEG compression that operates on $8 \times 8$-DCT blocks, a watermarking that operates on the same block size yields better robustness than that on the whole image [10].

The DCT allows an image to be divided into different frequency subbands: low frequency, middle frequency, and high frequency (see Figure 1). Embedding the watermark into the low-frequency subbands coefficient can degrade the image quality, whereas high frequency components are easily discarded after low pass filtering or JPEG compression. Therefore, for balancing the image fidelity and robustness, most watermarking techniques embed the watermark into the middle-frequency subbands coefficients.

\section{$5 \quad$ The Proposed Method}

We present an asymmetric watermarking technique for still images based on permutation using RC4 algorithm and chaotic map. There are three stages in this technique: the watermark generating, the watermark embedding, and the watermark detection. Each stage will be explained in following sub-sections.

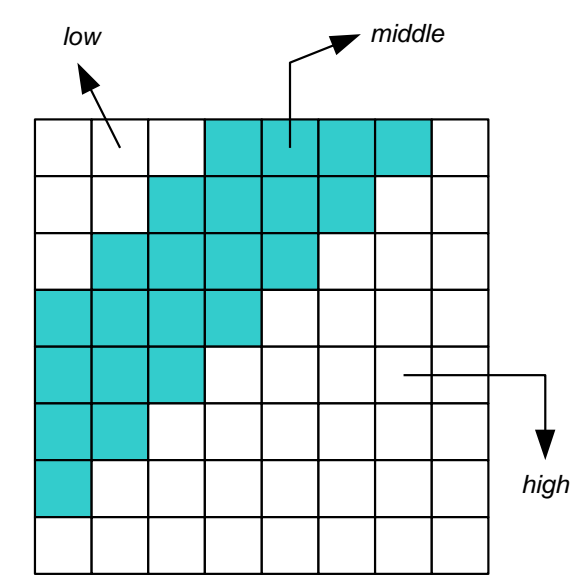

Figure 1 Definition of DCT regions. 


\subsection{Generation of Watermark}

The watermark size is about $25 \%$ of the original image size. Therefore, if the image size is $N_{1} \times N_{2}$ pixel, then watermark size is about $N_{1} N_{2} / 4$. The watermark is a sequence of real numbers chosen according to a normal distribution with mean $=0$ and variance $=1$ (notation: $\mathrm{N}(0,1)$ ).

Firstly, generate a public watermark $\mathbf{w}_{\mathbf{p}}$ whose length is $N$ according to normal distribution $\mathrm{N}(0,1)$ :

$$
\mathbf{w}_{\mathbf{p}}=\left(w_{p}(1), w_{p}(2), \ldots, w_{p}(N)\right)
$$

Next, we construct a secret watermark $\mathbf{w}_{\mathbf{s}}$ by permutating $\mathbf{w}_{\mathbf{p}}$ according to permutation table $S$ that is taken from RC4 algorithm (see Ssection 3):

$$
\begin{aligned}
\mathbf{w}_{\mathrm{s}} & =\left(w_{s}(1), w_{s}(2), \ldots, w_{s}(N)\right) \\
& =\left(w_{p}(S(1)), w_{p}(S(2)), \ldots, w_{p}(S(N))\right)
\end{aligned}
$$

The public watermark is published, but the permutation table $S$ and the initial value of the logistic map must be kept secret.

\subsection{Embedding of Watermark}

The watermark embedding scheme is combination of [11] and [10] schemes. The embedded watermark is combination of the secret and public watermark. This watermark is computed by formula [11]:

$$
\mathbf{w}_{\mathbf{e}}=(1-\alpha) \mathbf{w}_{\mathbf{s}}+\alpha \mathbf{w}_{\mathbf{p}}
$$

where the term $\alpha$ is a weighted factor to control the public detection threshold, and $0<\alpha<1$.

The original image $I$ is divided into small blocks of $8 \times 8$ pixel. Next, apply the DCT for every block, and then the DCT coefficients of the block -except DC value- are scanned by zigzag order to extract mid-frequency components. Suppose the selected components is represented by $\mathbf{f}$, then the embedded watermark $\mathbf{w}_{\mathrm{e}}$ is inserted into $\mathbf{f}$ by formula [10]:

$$
f_{w}(i)=f(i)+\gamma|f(i)| w_{e}(i)
$$

where $\gamma$ is a watermark strength constant that is adjusted to make the watermark imperceptible. Finally, using IDCT (inverse of the DCT), we get the watermarked image. 


\subsection{Watermark Detection}

The proposed detection technique does not require the original image and the secret watermark. Detector requires only the public watermark that has a correlation with the secret watermark. Given a received image and a public watermark, only two cases are possible: the image contains the watermark or the image does not contain the watermark.

Watermark detection is performed in the following steps. Firstly, the received image is divided into small blocks of $8 \times 8$ pixel. Next, apply the DCT for every block and then the DCT coefficients of the block, except DC value, are scanned by zigzag ordering to extract mid-frequency components. Suppose the selected component is represented by $\mathbf{f}^{*}$, then the correlation between $\mathbf{f}^{*}$ and the public watermark $\mathbf{w}_{\mathbf{p}}$ is computed by formula:

$$
c=\frac{1}{N} \sum_{i=i}^{N} f *(i) \cdot w_{p}(i)
$$

This correlation is compared to a threshold $T$ : if $|c|>T$, we say a watermark signal exists; otherwise, a watermark signal does not exist. The threshold $T$ is derived empirically by examining the correlation of random sequences.

\subsection{Security Analysis}

If an attacker wants to remove the watermark from the watermaked image, he (or she) does it by manipulating equation (4) to get $f(i)$ as follows:

$$
f(i)=\frac{f_{w}(i)}{1 \pm w_{e}(i)}
$$

The attacker knows $f_{w}(i), \mathbf{w}_{\mathbf{p}}$, and $\gamma$, but he (or she) must have $\mathbf{w}_{\mathbf{s}}$ in order to compute $w_{e}$ by using equation (5). Because $\mathbf{w}_{\mathbf{s}}$ is obtained by a secret permutation $S$, whereas $S$ also depends on a secret chaotic sequence, then the attacker cannot calculate (8). If the attacker tries to generate the chaotic sequence, he (or she) must try all possibility of initial values of the logistic map. Since the logistic map is sensitive to initial value, the attacker will fail to discover the chaotic sequence; in other words, it is impossible for the attacker to deduce the private watermark from the public information.

\section{$6 \quad$ Experiments and Results}

We program the watermarking algorithm using MATLAB 7. The test image is a $256 \times 256$ gray image 'peppers'. The public watermark is a $128 \times 128$ real 
matrix that has a normal distribution with mean $=0$ and variance $=1$. A chaotic sequence is generated by logistic map with the initial value $x_{0}=0.45$. We use $\alpha$ $=0.4$ and $\gamma=0.6$. And 500 random watermarks were generated for evaluating detection method.

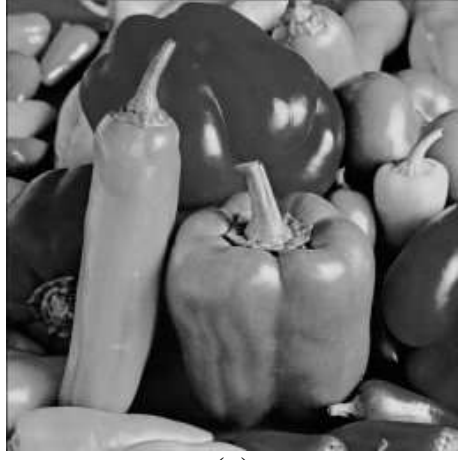

(a)

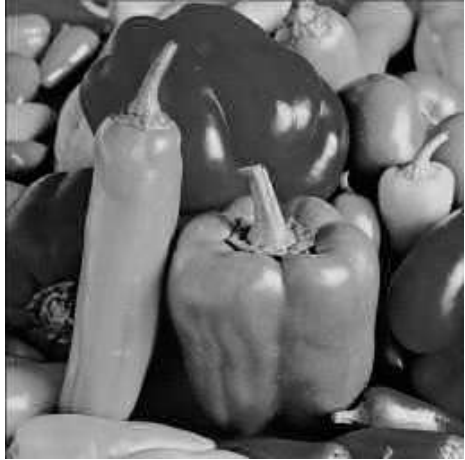

(b)

Figure 2 (a) Original image. (b) Watermarked image.

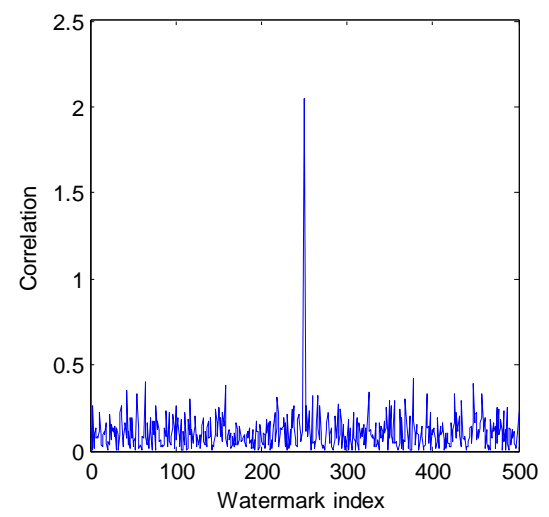

(a)

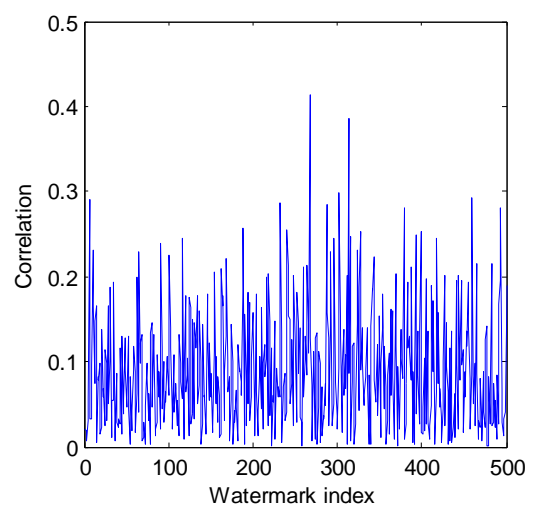

(b)

Figure 3 Detection threshold experimently; (a) case watermark exists, and (b) case no watermark exist.

Figure 2(a) shows the original image and Figure 2(b) shows the watermarked image $(P S N R=37.8044)$. Next, we derive the detection threshold empirically. Figure 3(a) shows the detection threshold of 500 random public watermarks studied. Only one public watermark, which has a correlation with the secret watermark, has a significantly higher correlation output than the others. In case of no attack, the detector result is $c=2.0507$. 
If the received image does not contain the watermark (in this experiment we use an unwatermarked 'peppers' image as input to detector), we get $c=0.1299$ and there is not a significantly higher correlation output than the others (Figure 3(b)). We conclude that the image does not contain the watermark.

We have tested robustness of the proposed technique againts various attacks using common image processings (JPEG compression, cropping, resizing, etc). We use Jasc Paint Shop version 6.01 as image processing software. For every attack, we set different thresholds. The experiment and results are explained as follows.

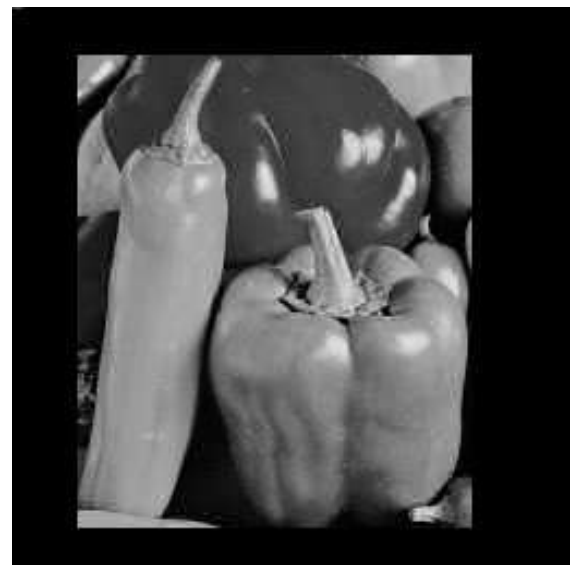

(a)

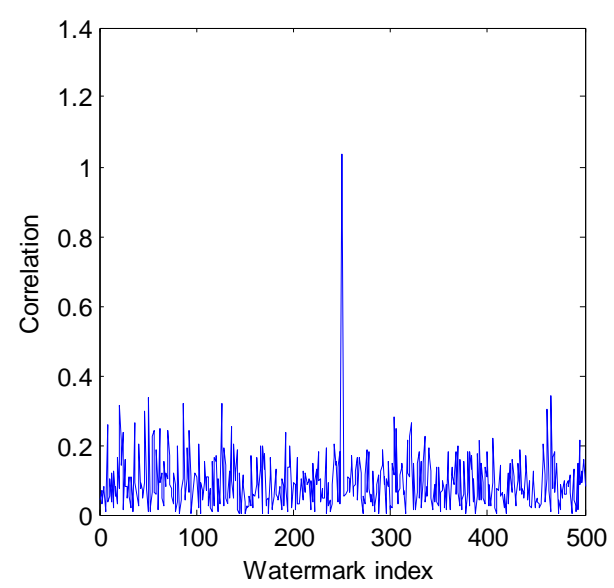

(b)

Figure 4 Cropping image. (b) Detector response. The watermark still can be detected.

\subsubsection{Experiment 1: Cropping}

Image cropping will remove some watermark information. In our simulation, we cut unimportant part from the watermarked image, the missing part of the image is replaced with black pixels (see Figure 4a). When the watermarked image is cropped, the correlation value is decreased $(c=1.0339)$ but this value is still significantly higher than the others (see Figure $4 \mathrm{~b}$ ). And we can see that the watermarking is robust against image cropping.

\subsubsection{Experiment 2: JPEG Compression}

We tested the robustness against JPEG compression with various compression qualities: $80 \%, 20 \%$, and $10 \%$ (extreme quality). In this experiment we use MATLAB to get JPEG file. To detect the watermark, the JPEG files are converted to the bitmap versions. The correlation values are decreased (Figure 
5). However, the correlation values are still above the random and we conclude the watermark is robust against JPEG compression.

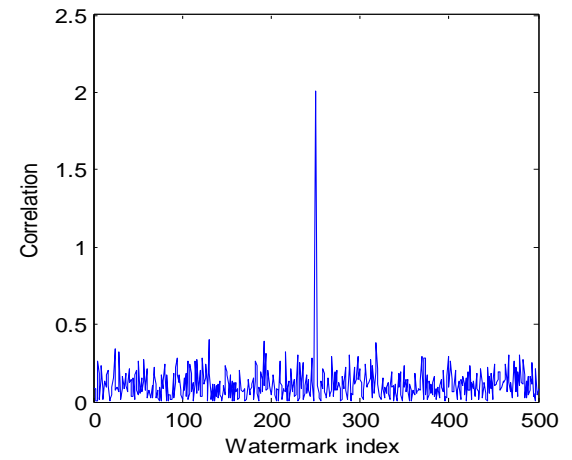

(a) Quality $80 \%$

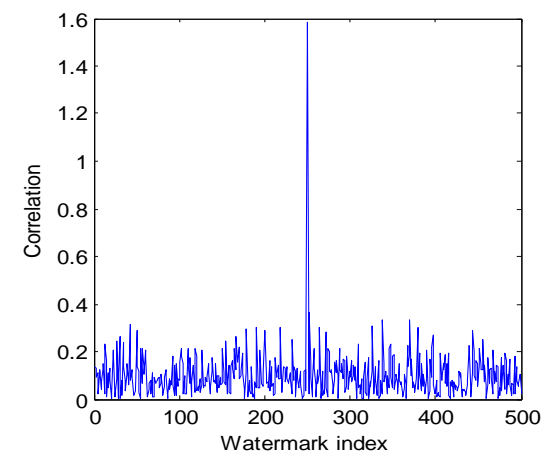

(b) Quality 20\%

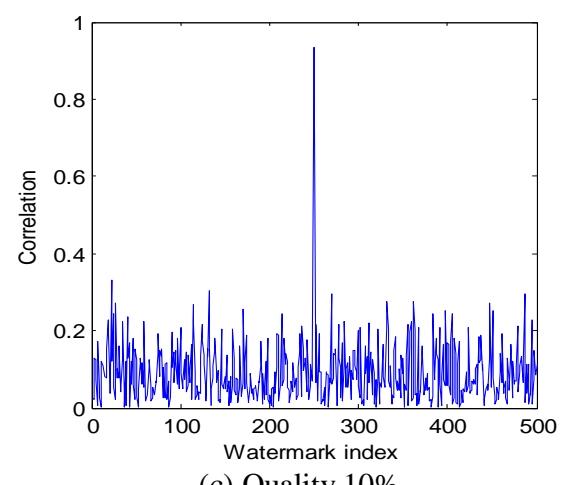

(c) Quality $10 \%$

Figure 5 JPEG compression with various qualites. The watermark still can be detected.

\subsubsection{Experiment 3: Sharpening and Adding Noise}

The watermarked image is sharpened until their edges look sharper than the original version. The detector shows that the correlation value is increased ( $c=$ 9.3838) and it is significantly above random (see Figure 6). We also add some noises like salt and peppers of $10 \%$. The results show that the watermark can be detected (see Figure 7, $c=1.9302$ ). We conclude from these experiments that the watermarking is robust againts image sharpening and noise. 


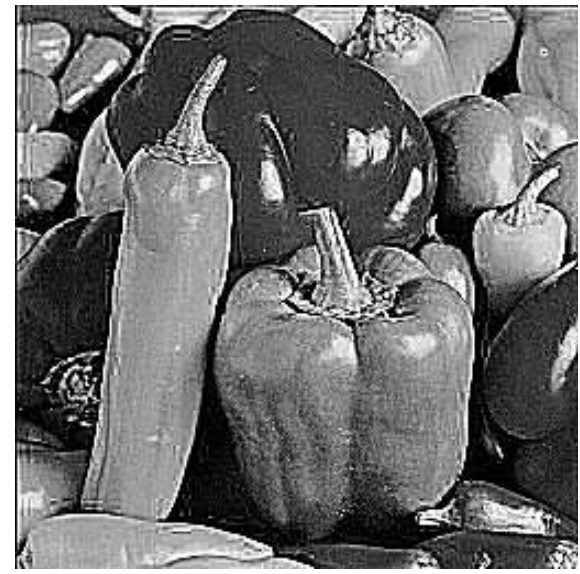

(a)

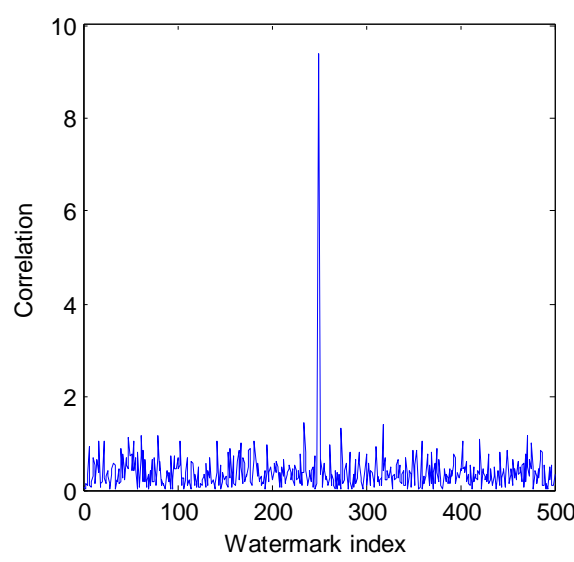

(b)

Figure 6 (a) Image sharpening. (b) Detector response.

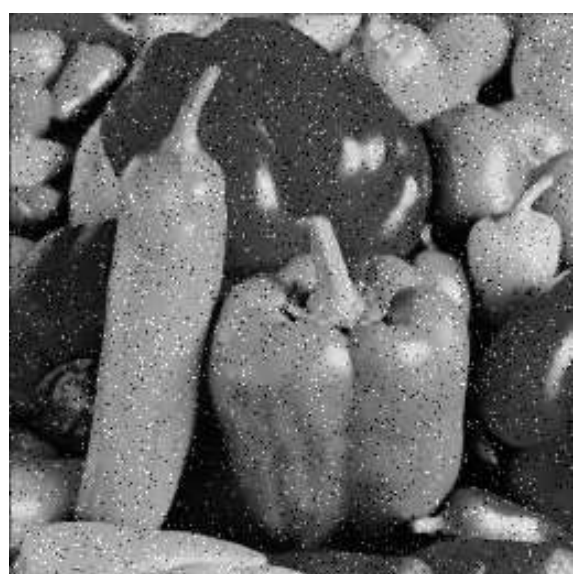

(a)

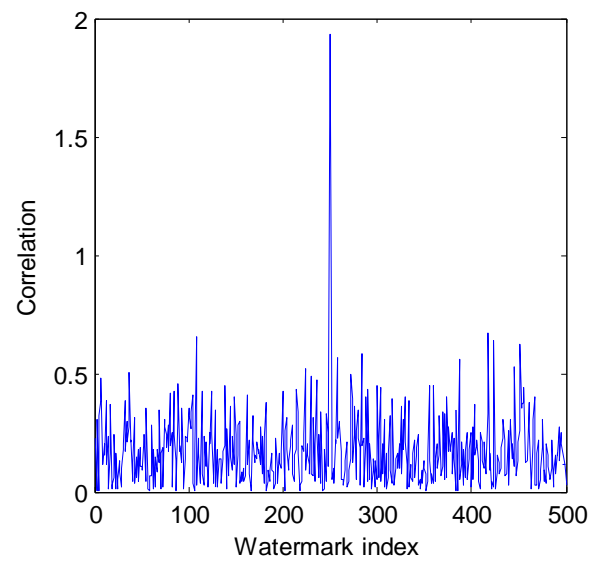

(b)

Figure 7 (a) Adding noise. (b) Detector response.

\subsubsection{Experiment 4: Rotation}

When an image is rotated, the DCT coefficient alignment changes. It causes the watermark undetectable. In order to the watermark can be detected, the rotated image must be returned to original position. In this experiment the watermarked image is rotated by as many as $10^{\circ}$ (Figure 8 ), then to detect the watermark, the rotated image is returned to the original position. It turns out that the correlation value is still above random $(c=1.09)$ and we conclude that the watermark can be detected. 

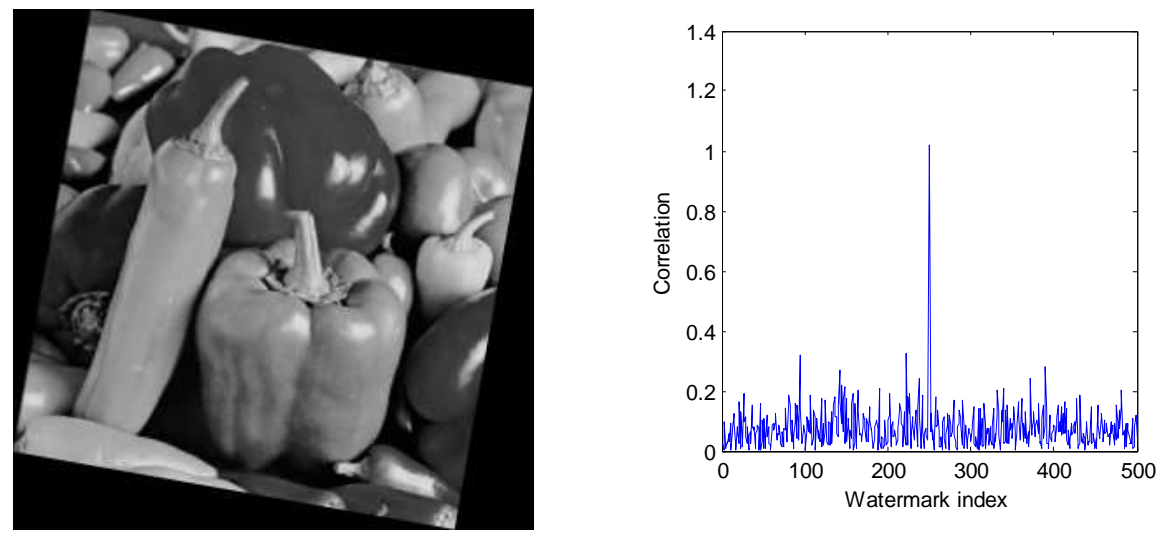

Figure 8 Image rotation with angle $10^{\circ}$. The watermark still can be detected.

\subsubsection{Experiment 5: Resizing}

The image watermarked is resized $75 \%, 50 \%$, and $200 \%$ of the original sizes respectively. To detect the watermark, the new image is returned to original size. We can see that the correlation values are still significantly higher than the others. We conclude that the watermarking is robust against image resizing.

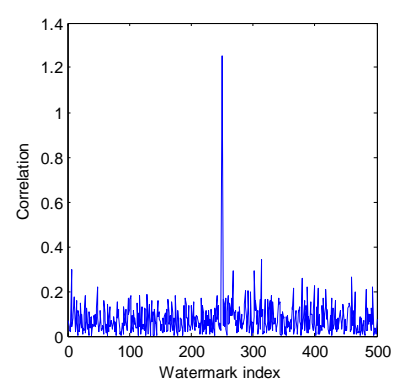

(a) Resizing $75 \%$

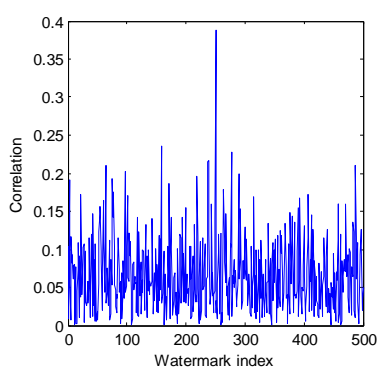

(b) Resizing $50 \%$

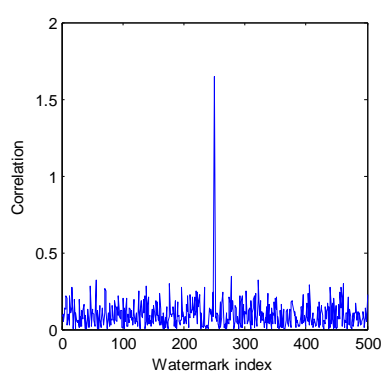

(c) Resizing $200 \%$

Figure 9 Image resizing $75 \%$. The watermark still can be detected.

\section{$7 \quad$ Conclusion}

In this paper an asymmetric watermarking technique for still images based on permutation-RC4 and chaotic map has been proposed. This technique applies the DCT to image blocks with $8 \times 8$ pixel and embed watermark into midfrequency components. The private watermark is produced by permutating the public watermark according to a part of RC4 algorithm and logistic map. The detection process is implemented by correlation test between public watermark 
and features of the image received. Simulation has confirmed that this technique is robust against non-malicious attacks (cropping, JPEG compression, resizing, rotation, sharpening, and noising).

\section{References}

[1] Cox, Ingemar J., et al., Secure Spread Spectrum Watermarking for Multimedia, IEEE Trans. On Image Processing, Vol. 6, No. 12, pp.16731687, Dec 1997.

[2] Agung, I. Wiseto P., Watermarking and Content Protection for Digital Images and Video, thesis of $\mathrm{PhD}$ in University of Surrey, 2002.

[3] Barni, Mauro \& Bartolini, Franco, Watermarking Systems Engineering, Marcel Dekker Publishing, 2004.

[4] Eggers, Joachim J., Su, Jonathan K. \& Girod, Bernd, Asymmetric Watermarking Schemes, GMD Jahrestagung, Proceddings, SpringerVerlag, 2000.

[5] Kim, T.T., Kim, T. \& Choi, H., Correlation-Based Asymmetric Watermarking Detector, Int. ITCC, 2003.

[6] Dawei, Zhao, Guanrong, Chen, Wenbo, Liu, A Chaos-Based Robust Wavelet-Dmain Watermarking Algorithm, Journal Chaos Solitons and Fractals, 22, 47-54, 2004.

[7] Fu, Yong-Gang, Shen, Rui Min \& Shen, Li Ping, A Novel Asymmetric Watermarking Scheme, Proc. of the $3^{\text {rd }}$ Int. Conference on Machine Learning and Cybernetics, 2004.

[8] Gui, Guo Fu, Jiang, Ling Ge \& He, Chen, A New Asymmetric Watermarking Scheme for Copyright Protection. IECE Trans. Fundamentals, Vol. E89-A, No. 2, February 2006.

[9] Wang, Hongxia, He, Chen \& Ding, Ke, Public Watermarking Based on Chaotic Map, IEICE Trans. Fundamentals, Vol. E87-A, August 2004.

[10] Jeong, Sangoh \& Hong, Kihyun, Dual Detection of A Watermark Embedded in the DCT Domain, EE368A Project Report, 2001.

[11] Gui, G.F., Jiang, L.G., He, C., General Construction of Asymmetric Watermarking Based on Permutation, Proc. IEEE Int. Workshop VLSI Design \& Video Tech., May 28, 2005. 